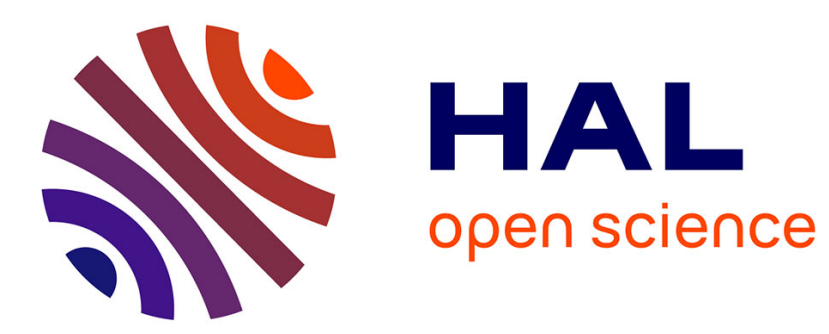

\title{
Toward a general procedure for extracting templates from chaotic attractors bounded by high genus torus
}

Martin Rosalie, Christophe Letellier

\section{To cite this version:}

Martin Rosalie, Christophe Letellier. Toward a general procedure for extracting templates from chaotic attractors bounded by high genus torus. International journal of bifurcation and chaos in applied sciences and engineering , 2014, 24 (4), pp.1450045. 10.1142/S021812741450045X . hal-01004483

\section{HAL Id: hal-01004483 \\ https://hal.science/hal-01004483}

Submitted on 5 Sep 2016

HAL is a multi-disciplinary open access archive for the deposit and dissemination of scientific research documents, whether they are published or not. The documents may come from teaching and research institutions in France or abroad, or from public or private research centers.
L'archive ouverte pluridisciplinaire HAL, est destinée au dépôt et à la diffusion de documents scientifiques de niveau recherche, publiés ou non, émanant des établissements d'enseignement et de recherche français ou étrangers, des laboratoires publics ou privés. 


\title{
Toward a general procedure for extracting templates from chaotic attractors bounded by high genus torus
}

\author{
Martin ROSALIE and Christophe LETELLIER \\ CORIA UMR-6614, Université de Rouen, Avenue de l'Université \\ Saint-Etienne-du-Rouvray, 76801, France \\ martin.rosalie@coria.fr
}

Received May 28, 2013(to be inserted by publisher)

\begin{abstract}
Topological analysis of chaotic attractor by the mean of template is rather well established for simple attractors like those solution to the Rössler system. Lorenz-like attractors are already slightly more complicated because they are bounded by a genus- 3 bounding torus, implying the necessity to use a two-component Poincaré section. In this paper, we enriched the concept of linking matrix to correctly describe algebraically template for attractor with $(g-1)$ components Poincaré section and whose bounding torus has $g$ interior holes aligned. An example with $g=5$ - a multispiral attractor - is explicitly treated.
\end{abstract}

Keywords: Topological analysis, attractor, template

\section{Introduction}

Chaos is now observed in many different fields like physics, astrophysics, astronomy, biology, chemistry, but it remains a challenge to have a conclusive proof for chaos from experimental data, mainly because establishing the existence of an underlying determinism is not trivial [Glass, 1999, 2009]. Once such a proof is obtained, a positive Lyapunov exponent can provide an additional characteristics of the dynamics [Wolf et al., 1985; Eckmann et al., 1986], but such a number is not very discriminative because it is too dependent on the sampling rate, the amplitude of the oscillation, etc. Moreover, there is no useful information in the largest Lyapunov exponent which can help to understand the deep difference between, for instance, a spiral attractor solution to the Rössler system [Rössler, 1976b], and the Lorenz attractor [Lorenz, 1963]. In these two examples, a topological analysis was performed in the original papers leading to a branched manifold (a template) which was named "blender" by [Rössler, 1976a] and "isopleths" by [Lorenz, 1963]. From a mathematical point of view, templates were considered by [Williams, 1977] and viewed as knot-holders by [Birman \& Williams, 1983]. Periodic orbits thus became central in the topological analysis as first used by [Poincaré, 1899]. Today, a topological analysis is required to be able to qualify an attractor as "new" or not [Letellier \& Aguirre, 2012].

Building the branched manifold is not necessarily the last objective since an algebraic description of template can be developed using linking matrices that encode most, if not all, topological properties synthesized by template [Tufillaro et al., 1992; Gilmore \& Lefranc, 2002]. Linking matrices were not too often used by mathematicians, perhaps because they mainly consider the Lorenz attractor which is not easily described by a linking matrix as we will detail in this paper. Indeed, the key step to describe attractors by linking matrices is to recognize that a Poincaré section can have multiple components, a property early 
introduced by [Letellier \& Gouesbet, 1996; Letellier et al., 1996] and later well justified in the context of bounding tori [Tsankov \& Gilmore, 2003, 2004]. We will thus show that there is, in fact, one linking matrix per component in the Poincaré section.

The subsequent part of this paper is organized as follows. Section 2 is devoted to the topology of the spiral attractor solution to the Rössler system. This section is the opportunity to introduce many concepts whose few are new and required for attractors characterized by multiple component Poincaré section. Section 3 discusses the case of the Lorenz attractor which is associated with a two-components Poincaré section. This latter case is a useful example to allow us to detail, in a still simple case, the necessity to enrich the concept of linking matrix. Section 4 is devoted to the example of a multispiral attractor bounded by a genus- 5 torus and thus characterized by a four-components Poincaré section. Section 5 sums up our procedure and gives some conclusions.

\section{The genus-1 spiral attractor}

\subsection{The Rössler system}

There are three levels to describe the topology of an attractor: i) the bounding torus, ii) the template and iii) the unstable periodic orbits. We will introduce these concepts in the case of the spiral attractor solution to the Rössler system [Rössler, 1976b]. The Rössler equations

$$
\left\{\begin{array}{l}
\dot{x}=-y-z \\
\dot{y}=x+a y \\
\dot{z}=b+z(x-c)
\end{array}\right.
$$

produce a chaotic attractor (Fig. 1) for parameter values $a=0.43293, b=2$, and $c=4$. This system has two singular points

$$
S_{ \pm}=\left\{\begin{array}{l}
x_{ \pm}=\frac{c \pm \sqrt{c^{2}-4 a b}}{2} \\
y_{ \pm}=\frac{-c \mp \sqrt{c^{2}-4 a b}}{2 a} \\
z_{ \pm}=\frac{c \pm \sqrt{c^{2}-4 a b}}{2 a} .
\end{array}\right.
$$

There are both saddle-focus points. Point $S_{-}$has a two-dimensional unstable manifold and is surrounded by the attractor. Point $S_{+}$has a two-dimensional stable manifold which is associated with the boundary of the attraction basin.

\subsection{Bounding torus and Poincaré section}

The spiral attractor can be bounded by a semi-permeable surface which is a genus-one torus [Tsankov \& Gilmore, 2003]. There is a canonical form to represent this bounding torus.

Definition 2.1. [Tsankov \& Gilmore, 2003] A bounding torus can be projected onto a two-dimensional planar surface in such a way that the projection is a disk with $g$ interior holes and that the flow on the exterior boundary and on these interior holes is in the same direction. By convention, the flow is clockwise. This is the canonical form of a bounding torus.

Any canonical form with $g \geq 3$ of a bounding torus is defined by $m$ circles and $n$ polygons, with $m+n=g$ and $m>n \geq 1$. The main departure between circles and polygons is that the latter can tear a branch into two sub-branches, an action that the formers cannot, as we will discuss later. The combination $(m, n)$ specifies the canonical in an unique way when $g<7$; for larger genus $g$, degeneracies occur [Tsankov \& Gilmore, 2004].

The spiral attractor is bounded by a genus-one torus whose canonical form is shown in Fig. 2. To have the spiral attractor visited clockwise, we applied a rotation $\mathrm{R}_{y}(\pi)$, that is, $(x, y, z) \mapsto(-x, y,-z)$ 


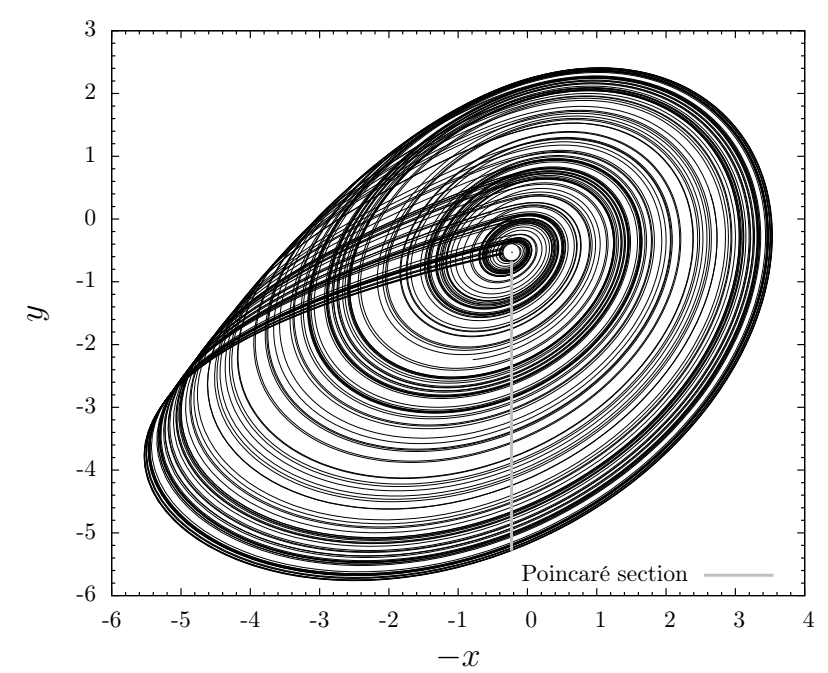

Fig. 1. Chaotic spiral attractor solution to the Rössler system (1). Parameter values $a=0.43293, b=2$, and $c=4$.

which does not modify the topology of the attractor. The interior circle of the bounding torus surrounds the saddle-focus point $S_{-}$. Note that there is not always a singular point associated with each hole of the bounding torus since, for instance, there are chaotic attractors bounded by genus-one torus and which are solution to systems without any singular point [Jafari et al., 2013].

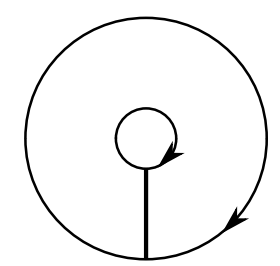

Fig. 2. Canonical form of the bounding torus associated with the spiral attractor Fig. 1. The Poincaré section is made of a single component.

The first information provided by the canonical form of bounding torus is the number of components the Poincaré section must have: an attractor bounded by a genus- $g(g \geq 3)$ torus is characterized by a Poincaré section with $g-1$ components. To genus-one bounding torus corresponds a single component Poincaré section. Components of the Poincaré section link the interior circles and the exterior boundary. Consequently, the spiral attractor can be studied using a single component Poincaré section defined as

$$
\mathcal{P} \equiv\left\{\left(y_{n},-z_{n}\right) \in \mathbb{R}^{2} \mid-x_{n}=-x_{-},-\dot{x}_{n}<0\right\},
$$

where $x_{-}$is the $x$-coordinate of the singular point $S_{-}$.

As introduced in [Gilmore \& Letellier, 2007], it is possible to introduce a normalized coordinate $\rho_{n}$ corresponding to the $n$th intersection with the Poincare section: $\rho_{n}$ is normalized to the unit interval, and oriented from the interior circles to the exterior boundary. The first-return map of a genus-one attractor is thus within the unit square (Fig. 3). As introduced by Poincaré, a first-return map allows to link the $n$th intersection to the $(n+1)$ th intersection. For chaotic attractors, the first-return map allows to define a partition between topologically inequivalent sub-spaces. In the case of a smooth unimodal first-return map as obtained for the spiral attractor, the increasing (decreasing) monotonic branch is order preserving (reversing) and, consequently, is associated with a branch presenting an even (odd) number of half-turns ( $\pi$-twists) [Letellier et al., 1995]. Let us label these two monotonic branches by "0" and "1", respectively, the parity of these symbols being related to the parity of the number of half-turns the corresponding branches present [Letellier et al., 1995]. 


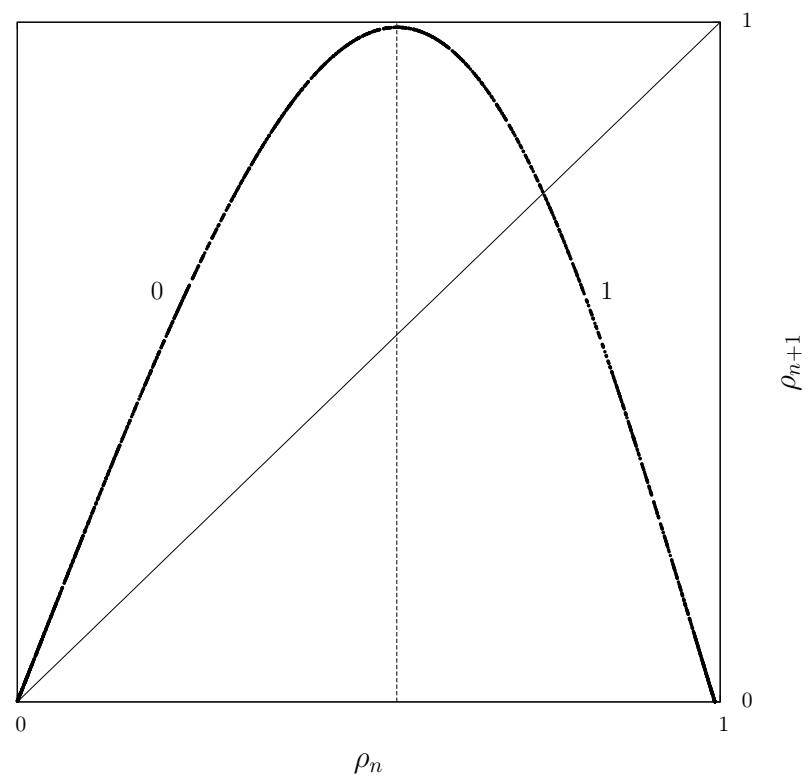

Fig. 3. First-return map to a one-component Poincaré section of the chaotic spiral attractor solution to the Rössler equations (1). Parameter values are those used for Fig. 1.

\subsection{Symbolic dynamics}

It is thus possible to convert any trajectory into a sequence of symbols according to

$$
a_{n}=\mid \begin{array}{lll}
0 & \text { if } & \rho_{n}<\rho_{c} \\
1 & \text { if } & \rho_{n}>\rho_{c}
\end{array}
$$

where $\rho_{c}=0.54$ is the ordinate of the critical point (the ordinate of the smooth maximum) of the first-return map shown in Fig. 3. The sequence of symbols thus obtained defines a symbolic dynamics, that is, infinite or bi-infinite sequences defined by a shift-invariant constant on the finite-length sub-sequences [Hao, 1989].

Definition 2.2. [Ghrist et al., 1997] Let $\alpha=\left\{a_{1}, \ldots, a_{N}\right\}$ be a set of $N$ symbols. Denote by

$$
\Sigma_{N}=\left\{\ldots a_{-2} a_{-1} a_{0} a_{1} a_{2} \ldots: a_{i} \in \alpha, \forall i \in \mathbb{Z}\right\}=\alpha^{\mathbb{Z}}
$$

the space of bi-infinite symbol sequences in $\alpha$. Points in $\Sigma_{N}$ are called trajectories. The shift map $\sigma: \Sigma_{N} \mapsto$ $\Sigma_{N}$ acts as

$$
\sigma\left(\ldots a_{-2} a_{-1} a_{0} a_{1} a_{2} \ldots\right)=\left(\ldots a_{-1} a_{0} a_{1} a_{2} a_{3} \ldots\right) .
$$

Any period- $p$ orbit embedded within the attractor is thus encoded by a periodic sequence of $p$ symbols, $p$ being the topological period of the orbit. For instance, orbit (1011) — parenthesis meaning that this is a periodic sequence infinitely repeated — is a period-4 orbit with three (one) intersections with the Poincaré section located in the decreasing (increasing) monotonic branch of the first-return map.

It is possible to defined a transition matrix.

Definition 2.3. [Ghrist et al., 1997] Given $T_{\sigma}$ an $N \times N$ matrix in zeros and ones, the subshift of finite type associated with $T_{\sigma}$ in the dynamical system $\left(\Sigma_{T}, \sigma\right)$ where $\Sigma_{T} \subset \Sigma_{N}$ is the set of admissible trajectories and $\sigma$ is the shift map. The matrix $T_{\sigma}$ is known as the transition matrix for $\Sigma_{T}$, since it specifies those transitions between symbols that are possible within a sequence.

In the case of the spiral attractor, as shown by the first-return map, the transition matrix is

$$
T_{\sigma}=\left[\begin{array}{ll}
1 & 1 \\
1 & 1
\end{array}\right] .
$$




\subsection{Template}

The topological properties of the attractor are synthesized by a branched manifold, named template.

Definition 2.4. (Adapted from [Ghrist et al., 1997]) A template is a compact branched two-manifold with boundary and smooth expansive semiflow built locally from three types of charts: splitting, tearing, merging and joining. Each chart (Figs. 4) carries a semiflow, endowing the template with an expanding semiflow, and gluing maps between charts must respect the semiflow and act linearly on the edges.

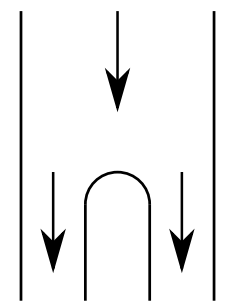

(a) Splitting chart

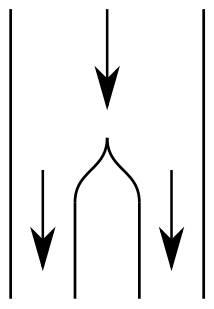

(b) Tearing chart

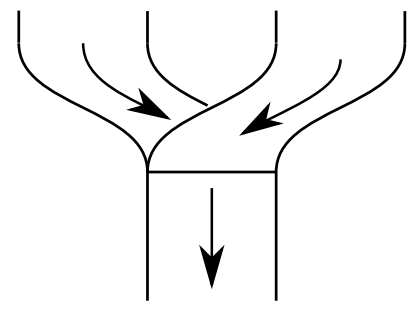

(c) Joining chart

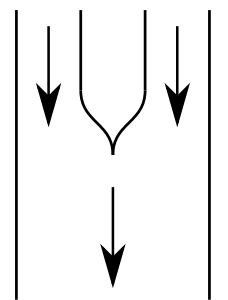

(d) Merging chart

Fig. 4. The different charts that can be used to draw a template.

A branch can virtually be split in two topologically inequivalent sub-branches to improve the readability of the template: it is virtual in the sense that the corresponding flow does not present such a split between the sub-branches. This process is represented by a "splitting chart". The edge of the splitting chart is associated with the critical point of the first-return map. A branch can be actually teared into two subbranches in the neighborhood of an interior polygon. Such a tearing can be indeed observed in the flow. This process is represented by a "tearing" chart (Fig. 4b). Sub-branches can be unified into a single branch according to a "joining" chart (Fig. 4c) which necessarily requires a stretching and squeezing mechanisms. To represent the joining chart, we use the standard joining convention introduced by [Tufillaro et al., 1992], and named by them standard insertion convention.

Definition 2.5. The standard convention to represent a joining chart is to squeeze the $N$ branches from back (left) to front (right).

An example of non-standard and standard joining convention is shown in Fig. 5. It should be noted that switching from a non-standard to a standard convention may induce supplementary permutations.

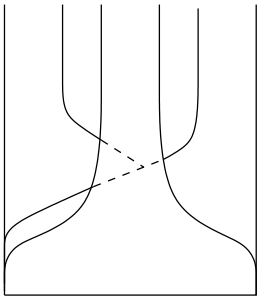

(a) Non-standard

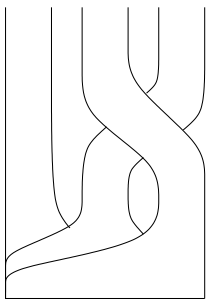

(b) Standard

Fig. 5. Examples of non-standard and standard joining chart [Tufillaro et al., 1992].

Rather than joined, one can also imagine that the $N$ branches are merged (Fig. $4 \mathrm{~d}$ ) as introduced in [Rosalie \& Letellier, 2013]. In this case, there is no mixing property as induced by the joining chart. Joining chart is therefore a required ingredient to produce chaotic behaviors. The main interest of the merging chart is that it helps us to understand that the mechanism responsible for the chaotic nature of a trajectory is encoded, from the template point of view, in the joining chart.

The template for the spiral attractor (Fig. 1) is made of one splitting chart, torsions and permutations applied to the $N=2$ branches, and one joining chart (Fig. 6). 


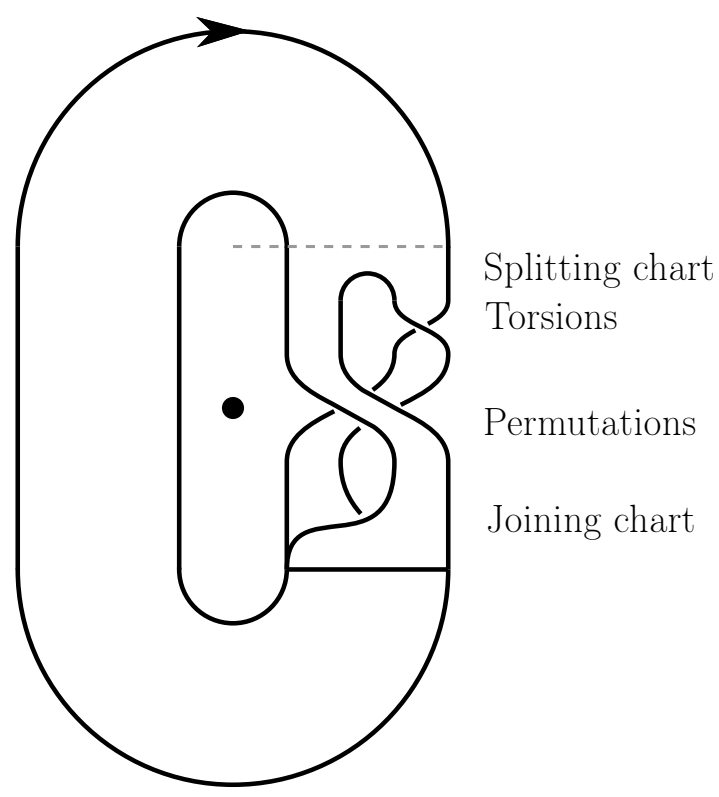

Fig. 6. Template of the spiral attractor (Fig. 1) solution to the Rössler system.

\subsection{Linker and linking matrix}

Definition 2.6. A linker is a sub-template at least describing torsions and permutations between the $N$ branches of the template, and possibly including splitting chart and tearing charts, and one merging or joining chart.

The linker must be represented with the branches ordered according to the order they appear in the first-return map built on variable $\rho_{n}$, that is, from the interior circle (left) to the exterior boundary (right).

Definition 2.7. A linker is said to be closed when it starts with a splitting or tearing chart and ends with one joining or merging chart. It is said inward (outward) open when there is no splitting nor tearing (no merging nor joining) chart. It is open when there is no chart, but only torsions and permutations between the $N$ branches (Figs. 7).

Proposition 1. Representing the attractor in a such a manner that the flow is clockwise as used for the canonical form of bounding torus, ordering the branches according to the first-return map and using the standard joining convention, the topology of a genus-one chaotic attractor is described by an unique closed mixer.

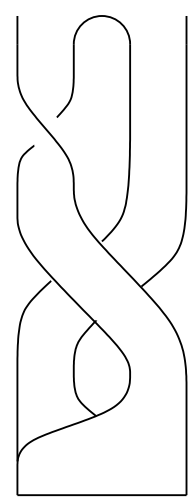

(a) Closed

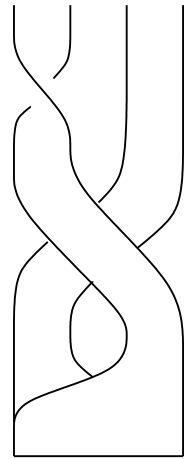

(b) Inward open

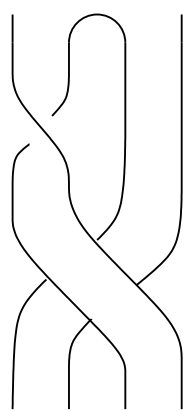

(c) Outward open

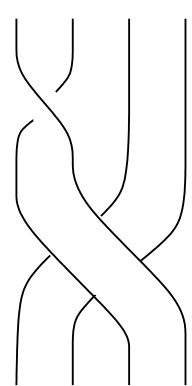

(d) Open

Fig. 7. Examples of close, semi-open and open linkers. 
Torsions and permutations applied to the $N$ branches of a linker are described by a linking matrix $L$.

Definition 2.8. A linking matrix $L$ is a $N \times N$ matrix of integers such as $L_{i i}$ is the signed number of half-turns applied to the $i$ th branch and $L_{i j}$ the signed number of permutations between the $i$ th and the $j$ th branches. Sign convention for torsions and permutations is shown in Fig. 8.

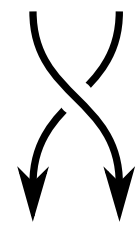

(a) Negative crossings

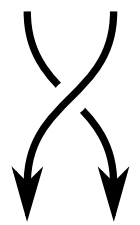

(b) Positive crossings

Fig. 8. Sign convention for torsions, permutations and crossings.

The linkers shown in Figs. 7 are described by the linking matrices as follows.

(1) Closed linker (Fig. 7a): $L_{\cap \mid}=\left[\begin{array}{cc}-1 & 0 \\ -1 & -1\end{array}\right]$ where the left bracket designates the splitting (or tearing) chart and the double right bracket the joining chart. When the joining chart is replaced with a merging chart, the linking matrix is thus $L_{\cap \cup}=\left[\begin{array}{cc}-1 & 0 \\ -1 & -1\end{array}\right]$, the right double bracket being replaced with the simple right bracket.

(2) Inward open linker (Fig. 7b): $L_{\mid}=\left|\begin{array}{cc}-1 & 0 \\ -1 & -1\end{array}\right|$, the left bar meaning that there is no splitting nor tearing chart.

(3) Outward open linker (Fig. 7c): $L_{\cap}=\left[\begin{array}{cc}-1 & 0 \\ -1 & -1\end{array} \mid\right.$, the right bar meaning that there is no joining nor merging chart.

(4) Open linker (Fig. 7d): $L=\left|\begin{array}{cc}-1 & 0 \\ -1 & -1\end{array}\right|$.

Definition 2.9. A mixer is a closed linker $\mathcal{L}_{\cap \mid}$ or an inward open linker $\mathcal{L}_{\mid}$ended by a joining chart.

Corollary 2.1. According to proposition 1, a genus-one chaotic attractor is described by a unique closed mixer, that is, by a unique closed linking matrix $L_{\cap}$.

The template for the spiral attractor is thus described by the linking matrix

$$
R=\left[\begin{array}{cc}
0 & -1 \\
-1 & -1
\end{array}\right] .
$$

\subsection{Linking numbers}

Linking numbers are topological invariants which can be counted in a regular plane projection of two periodic orbits $\mathcal{O}$ and $\mathcal{O}^{\prime}$ (here considered as knots): they are equal to the half-sum of the oriented "crossings", signed according to the convention shown in Fig. 8 [Tufillaro et al., 1992; Letellier et al., 1995]. From a linking matrix $L_{\cap !}$, it is possible to predict the linking number between two periodic orbits $\mathcal{O}$ and $\mathcal{O}^{\prime}$ [Le Sceller et al., 1994]. Let $\mathcal{O}$ and $\mathcal{O}^{\prime}$ be a period- $p$ and a period- $p^{\prime}$ orbits associated with the orbital sequences

$$
\alpha=a_{1} a_{2} \ldots a_{p}
$$

and

$$
\alpha^{\prime}=a_{1}^{\prime} a_{2}^{\prime} \ldots a_{p^{\prime}}^{\prime},
$$


respectively. The linking number between these two periodic orbits is

$$
\operatorname{Lk}\left(\mathcal{O}, \mathcal{O}^{\prime}\right)=\sum_{i=1}^{p} \sum_{j=1}^{p^{\prime}} L_{a_{i} a_{j}^{\prime}}+N_{\text {joining }}\left(\mathcal{O}, \mathcal{O}^{\prime}\right)
$$

where $N_{\text {joining }}\left(\mathcal{O}, \mathcal{O}^{\prime}\right)$ counts the number of oriented crossings occurring in the joining chart. It can be determined from $\alpha, \alpha^{\prime}$ and $L_{\cap}$ according to a procedure detailed in [Le Sceller et al., 1994] and whose details are out of the scope of this paper. In [Le Sceller et al., 1994], $N_{\text {joining }}$ was designated as $N_{\text {ramification }}$ but, since it is necessarily related to the joining chart, it turns to be better named by $N_{\text {joining }}$.

A template is validated when the linking numbers it predicts between pairs of periodic orbits are equal to those between the corresponding periodic orbits numerically extracted from the chaotic attractors [Tufillaro et al., 1992; Letellier et al., 1995; Gilmore \& Lefranc, 2002]. The specific case of the spiral Rössler attractor is detailed in [Letellier et al., 1995].

\section{The genus-3 Lorenz attractor}

Templates for some attractors bounded by genus-3 torus had already been established [Birman \& Williams, 1983; Kocarev et al., 1994; Letellier \& Gilmore, 2013]. They are much more difficult to handle than templates for genus-one attractor. In particular, their descriptions by linking matrices are not yet welldeveloped. Consequently, we will detail this aspect in the subsequent part of this paper, first with the help of the genus-3 Lorenz attractor.

The Lorenz differential equations [Lorenz, 1963]

$$
\left\{\begin{array}{l}
\dot{x}=\sigma(y-x) \\
\dot{y}=R x-y-x z \\
\dot{z}=-\beta z+x y,
\end{array}\right.
$$

produce a so-called Lorenz attractor (Fig. 9a) for parameter values $R=28, \beta=\frac{8}{3}$ and $\sigma=10$. This attractor is projected in the $x-y$ plane where the flow is clockwise. It is bounded by a genus- 3 torus, two interior circles — which can be associated with the two saddle-focus points

$$
S_{ \pm}=\mid \begin{aligned}
& x_{ \pm}= \pm \sqrt{b(R-1)} \\
& y_{ \pm}= \pm \sqrt{b(R-1)} \\
& z_{ \pm}=R-1
\end{aligned}
$$

and one interior polygon surrounding the saddle singular point located at the origin of the phase space (Fig. 9b).

The Lorenz equations are equivariant under a rotation symmetry $\mathcal{R}_{z}(\pi)$ which maps the left wing into the right wing, and vice versa. It was shown that the Poincaré section must have two components to properly compute the Lorenz map [Letellier et al., 1994], a property now well-explained by bounding tori [Tsankov \& Gilmore, 2004]. We will use in this work the two components defined as [Letellier et al., 1994]

$$
\mathcal{P} \equiv \mathcal{C}_{A} \cup \mathcal{C}_{B} \equiv\left\{\left(y_{n}, z_{n}\right) \in \mathbb{R}^{2} \mid x_{n}=x_{+}, \dot{x}_{n}<0\right\} \cup\left\{\left(y_{n}, z_{n}\right) \in \mathbb{R}^{2} \mid x_{n}=x_{-}, \dot{x}_{n}>0\right\} .
$$

where $x_{ \pm}$are the $x$-coordinates of the two symmetry related singular points $S_{ \pm}$. When the first-return map to the Poincaré section is computed with $\rho_{n}$ left in the unit interval ]0;1[, the Lorenz map is obtained [Letellier \& Gouesbet, 1996]. In order to develop a general method for characterizing attractors with multiple component Poincaré section, it is useful to distinguish the contribution of these different components. We thus used

$$
\rho_{n}=\mathbb{I}_{A} \cdot \rho_{A, n}+\mathbb{I}_{B} \cdot\left(1+\rho_{B, n}\right)
$$

where $\rho_{A, n}$ and $\rho_{B, n}$ are the $\rho$-coordinate of the $n$th intersection with the Poincaré section located in component $\mathcal{C}_{A}$ and $\mathcal{C}_{B}$, respectively, and where $\mathbb{I}_{\mathcal{C}}$ is the indicator function where

$$
\mathbb{I}_{\mathcal{C}}=\mid \begin{array}{ll}
0 & \text { if } x \notin \mathcal{C} \\
1 & \text { if } x \in \mathcal{C} .
\end{array}
$$




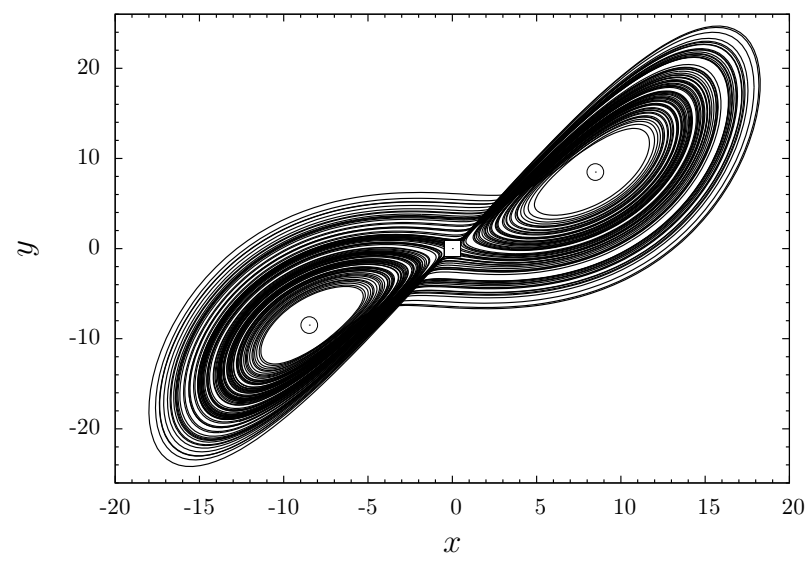

(a) The Lorenz attractor

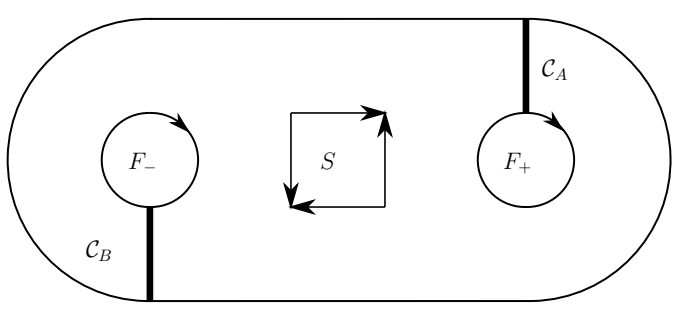

(b) Canonical genus-3 bounding torus

Fig. 9. Chaotic attractor solution to the Lorenz equations $(8)$ and its singular points $(\odot$ for saddle-focus points and $\odot$ for the saddle point). The canonical form of the corresponding bounding torus is also shown with the two components $\mathcal{C}_{A}$ and $\mathcal{C}_{B}$ of the Poincaré section. Parameter values $R=28, \beta=\frac{8}{3}$ and $\sigma=10$.

This was implicitly used by [Byrne et al., 2004]. Variable $\rho_{n}$ is thus in the range $] 0 ; 1[\cup] 1 ; 2[$. The corresponding first-return map is shown in Fig. 10: this is a four branch map. We labelled these branches

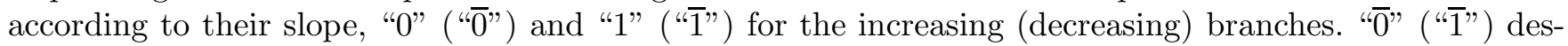
ignates the branch which is obtained by applying the rotation $\mathcal{R}_{z}(\pi)$ to branch " 0 " (" 1 "). The symbol transition matrix is

$$
T_{a}=\left[\begin{array}{llll}
1 & 1 & 0 & 0 \\
0 & 0 & 1 & 1 \\
0 & 0 & 1 & 1 \\
1 & 1 & 0 & 0
\end{array}\right] .
$$

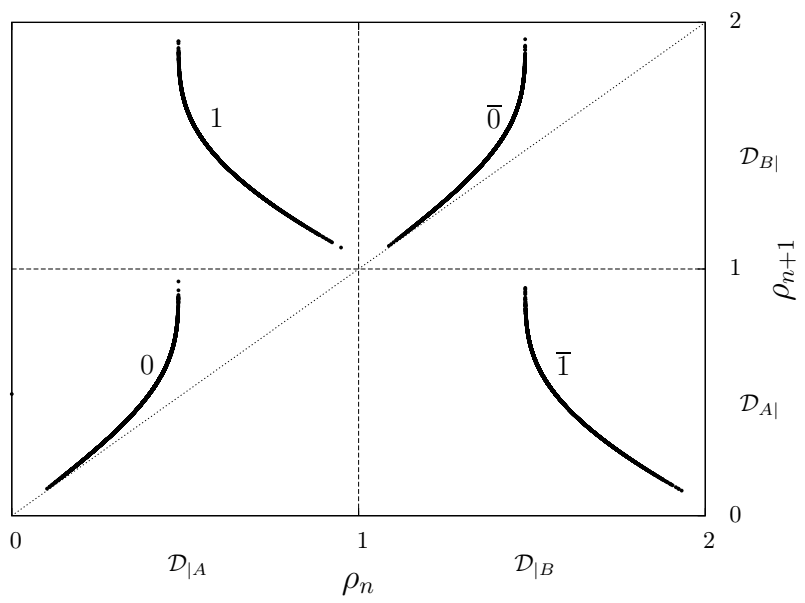

Fig. 10. First-return map to a two-component Poincaré section of the chaotic attractor solution to the Lorenz equations (8). Parameter values are those used for Fig. 9.

From the bounding torus (Fig. $9 \mathrm{~b}$ ), it is seen that the trajectory from $\mathcal{C}_{A}$ can go to $\mathcal{C}_{A}$ or $\mathcal{C}_{B}$, and from $\mathcal{C}_{B}$ to $\mathcal{C}_{B}$ with itself and $\mathcal{C}_{A}$. A transition matrix $T_{\mathcal{C}}$ between the components of the Poincaré section is thus

$$
T_{\mathcal{C}}=\left[\begin{array}{ll}
1 & 1 \\
1 & 1
\end{array}\right]
$$


It is possible to consider the Lorenz attractor from two points of view. The first one considers the attractor as made of two parts, one being associated with the branches " 0 " and " 1 " issued from component $\mathcal{C}_{A}$, the second part being the symmetric of the first and is made of branches " $\overline{0}$ " and " "1" arising from component $\mathcal{C}_{B}$. Let us designate these two parts as the two domains $\mathcal{D}_{\mid A}=\{0,1\}$ and $\mathcal{D}_{\mid B}=\{\overline{0}, \overline{1}\}$ where the subscript $\mid A(\mid B)$ means that the branches are coming from component $\mathcal{C}_{A}\left(\mathcal{C}_{B}\right)$. The second point of view is to consider the branches joined at a given component: we have thus $\mathcal{D}_{A \mid}=\{0, \overline{1}\}$ and $\mathcal{D}_{B \mid}=\{1, \overline{0}\}$ where $A \mid$ $(B \mid)$ means that the branches are joined at component $A(B)$. Since the mixing properties are induced by the joining chart, there is no other choice than using the second point of view to describe the attractor in terms of linkers (mixers).

Our objective is now to describe the topology of the Lorenz attractor by two mixers, each of them being associated with the domains $\mathcal{D}_{A \mid}=\{0, \overline{1}\}$ and $\mathcal{D}_{B}=\{1, \overline{0}\}$, respectively. A template proposed for the Lorenz attractor is shown in Fig. 11 [Letellier and Gilmore, 2001]. From that template, we can see that there is one inward open mixer associated with each component of the Poincaré section. Mixer $\mathcal{M}_{A \mid}\left(\mathcal{M}_{B \mid}\right)$ corresponds to domain $\mathcal{D}_{A \mid}=\{0, \overline{1}\}\left(\mathcal{D}_{B \mid}=\{1, \overline{0}\}\right)$. These mixers are described by the linking matrices

$$
\left.\left.M_{A \mid}=\mid \begin{array}{ll}
0 & 0 \\
0 & 1
\end{array}\right] \quad \text { and } \quad M_{B \mid}=\mid \begin{array}{ll}
0 & 0 \\
0 & 1
\end{array}\right],
$$

respectively. Matrices $M_{A \mid}$ and $M_{B \mid}$ describe torsions and permutations between branches " 0 " and "T" of domain $\mathcal{D}_{A \mid}$ and, branches " $\overline{0}$ " and " 1 " of domain $\mathcal{D}_{B \mid}$, respectively. Branches are ordered in the "natural" order they occurs in each of the components $\mathcal{C}_{A}$ and $\mathcal{C}_{B}$. Note that these two "natural" orders are the order that, for instance, branches "0" and " $\overline{1}$ " would have when they are drawn in the unit square using $\rho_{A, n}$ and $\rho_{B, n}$ (and not $\rho_{n}$ ). We have thus the natural order $0 \overline{1}(\overline{0} 1)$ in domain $\mathcal{D}_{A \mid}=\{0, \overline{1}\}\left(\mathcal{D}_{B \mid}\right)$.

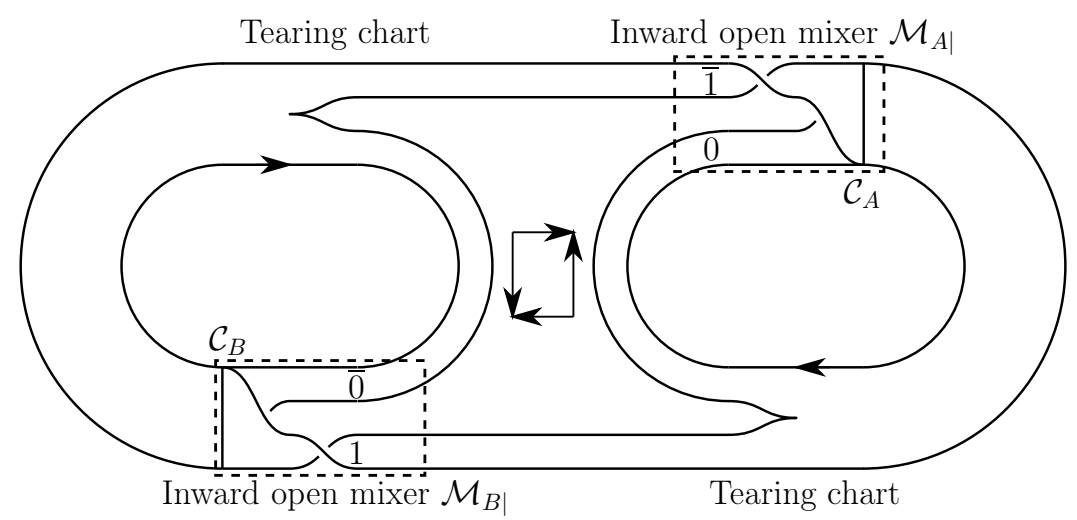

Fig. 11. Template that describes the Lorenz attractor shown in Fig. 9.

\section{The genus-5 multispiral attractor}

To show that our procedure is quite general, we will investigate now a genus-5 multispiral attractor produced by a set of differential equations proposed by [Aziz-Alaoui, 1999]

$$
\left\{\begin{array}{l}
\dot{x}=\alpha\left[y-x-f_{3}(x)\right] \\
\dot{y}=x-y+z \\
\dot{z}=-\beta x-\gamma y
\end{array}\right.
$$

where

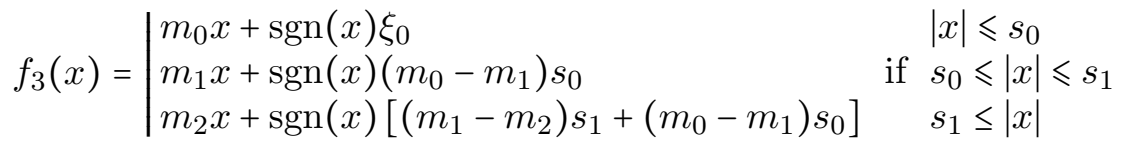


is a piecewise linear function. With parameter values $\alpha=14.6, \beta=12, \gamma=0.9, \xi_{0}=0, s_{0}=1, s_{1}=3$, $m_{0}=-5 / 7, m_{1}=-8 / 7$ and $m_{2}=-0.7$, the chaotic attractor obtained is projected in the $x$ - $y$ plane (Fig. 12). The system (16) has three saddle-focus singular points and two saddle singular points (also shown in Fig. 12). This system is equivariant under an inversion symmetry.

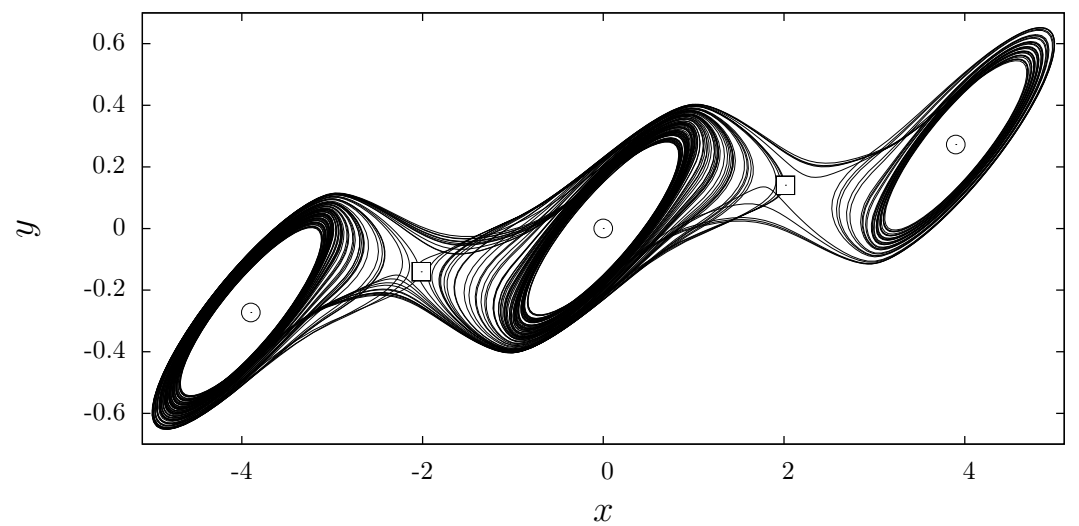

Fig. 12. Chaotic multispiral attractor solution to the equation (16). Parameter values $\alpha=14.6, \beta=12, \gamma=0.9, \xi_{0}=0, s_{0}=1$, $s_{1}=3, m_{0}=-5 / 7, m_{1}=-8 / 7$ and $m_{2}=-0.7$.

This attractor is bounded by a genus- 5 torus with three interior circles and two interior polygons. The Poincaré section is made of four components $\mathcal{C}_{A}, \mathcal{C}_{B}, \mathcal{C}_{C}$, and $\mathcal{C}_{D}$. The component transition matrix is

$$
T_{\mathcal{C}}=\left[\begin{array}{llll}
0 & 1 & 1 & 0 \\
0 & 1 & 1 & 0 \\
1 & 0 & 0 & 1 \\
1 & 0 & 0 & 1
\end{array}\right] .
$$

Under the inversion symmetry, component $\mathcal{C}_{A}\left(\mathcal{C}_{B}\right)$ is mapped into $\mathcal{C}_{C}\left(\mathcal{C}_{D}\right)$, and vice versa.

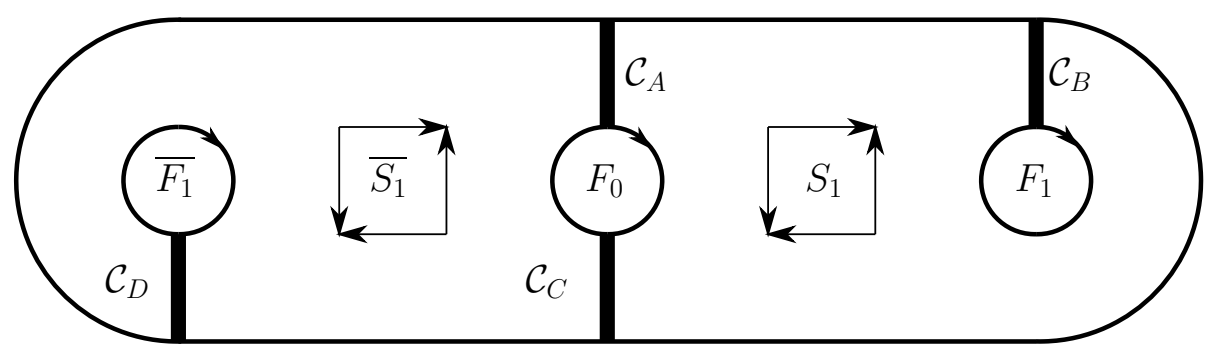

Fig. 13. Canonical form $A_{3}$ with the four-component Poincaré section $\mathcal{C}_{A}, \mathcal{C}_{B}, \mathcal{C}_{C}$ and $\mathcal{C}_{D}$.

The first-return map is built on variable

$$
\rho_{n}=\mathbb{I}_{A} \cdot \rho_{A, n}+\mathbb{I}_{B} \cdot\left(1+\rho_{B, n}\right)+\mathbb{I}_{C} \cdot\left(2+\rho_{C, n}\right)+\mathbb{I}_{D} \cdot\left(3+\rho_{D, n}\right),
$$

leading to a map plotted in a square whose side have four units in length (Fig. 14). We will distinguish twelve monotonic branches in this map, encoded according to their slope by $0,1,3,4,5,7, \overline{0}, \overline{1}, \overline{3}, \overline{4}$, $\overline{5}$, and $\overline{7}$. Symbols $\bar{a}$ are symmetry related to symbols $a$. Obtained from the first-return map, the symbol 
transition matrix

$$
T_{a}=\left[\begin{array}{llllllllllll}
0 & 0 & 0 & 0 & 0 & 0 & 1 & 1 & 1 & 0 & 0 & 0 \\
0 & 0 & 0 & 0 & 0 & 0 & 1 & 1 & 1 & 0 & 0 & 0 \\
0 & 0 & 0 & 1 & 0 & 0 & 0 & 0 & 0 & 0 & 0 & 0 \\
0 & 0 & 0 & 1 & 1 & 1 & 0 & 0 & 0 & 0 & 0 & 0 \\
0 & 0 & 0 & 1 & 1 & 1 & 0 & 0 & 0 & 0 & 0 & 0 \\
0 & 0 & 0 & 0 & 0 & 0 & 1 & 0 & 0 & 0 & 0 & 0 \\
1 & 1 & 1 & 0 & 0 & 0 & 0 & 0 & 0 & 0 & 0 & 0 \\
1 & 1 & 1 & 0 & 0 & 0 & 0 & 0 & 0 & 0 & 0 & 0 \\
0 & 0 & 0 & 0 & 0 & 0 & 0 & 0 & 0 & 1 & 0 & 0 \\
0 & 0 & 0 & 0 & 0 & 0 & 0 & 0 & 0 & 1 & 1 & 1 \\
0 & 0 & 0 & 0 & 0 & 0 & 0 & 0 & 0 & 1 & 1 & 1 \\
1 & 0 & 0 & 0 & 0 & 0 & 0 & 0 & 0 & 0 & 0 & 0
\end{array}\right] .
$$

We have four domains in the multispiral attractors defined as

$$
\begin{aligned}
& \mathcal{D}_{A \mid}=\{\overline{0}, \overline{1}, \overline{7}\}, \\
& \mathcal{D}_{B \mid}=\{4,5,3\}, \\
& \mathcal{D}_{C \mid}=\{0,1,7\}, \\
& \mathcal{D}_{D \mid}=\{\overline{4}, \overline{5}, \overline{3}\},
\end{aligned}
$$

where the branches are ordered according to the order they appear in each domain or, equivalently, in a single unit square using, for instance, variables $\rho_{A, n}$ for branch " 3 " and $\rho_{B, n}$ for branches " 4 " and " 5 " to order these three branches in the domain $\mathcal{D}_{B \mid}$. To be more explicit, let be the $n$th intersection in branch " 3 " and the $m$ th intersection in branch " 4 ". We have thus $\rho_{A, n}>\rho_{B, m}$, meaning that, when joined in component $\mathcal{C}_{B}$, branch 3 is closer to the exterior boundary than branch 4 . The orders specified for each domain are the "natural" orders to be used to order the periodic points in the corresponding component of the Poincaré section (see [Hao, 1989; Letellier et al., 1995] for details). The linking matrices must be written using these orders. From our symbol notations, it clearly appears that domain $\mathcal{D}_{A \mid}\left(\mathcal{D}_{B \mid}\right)$ is the symmetric of domain $\mathcal{D}_{C \mid}\left(\mathcal{D}_{D \mid}\right)$, and vice versa.

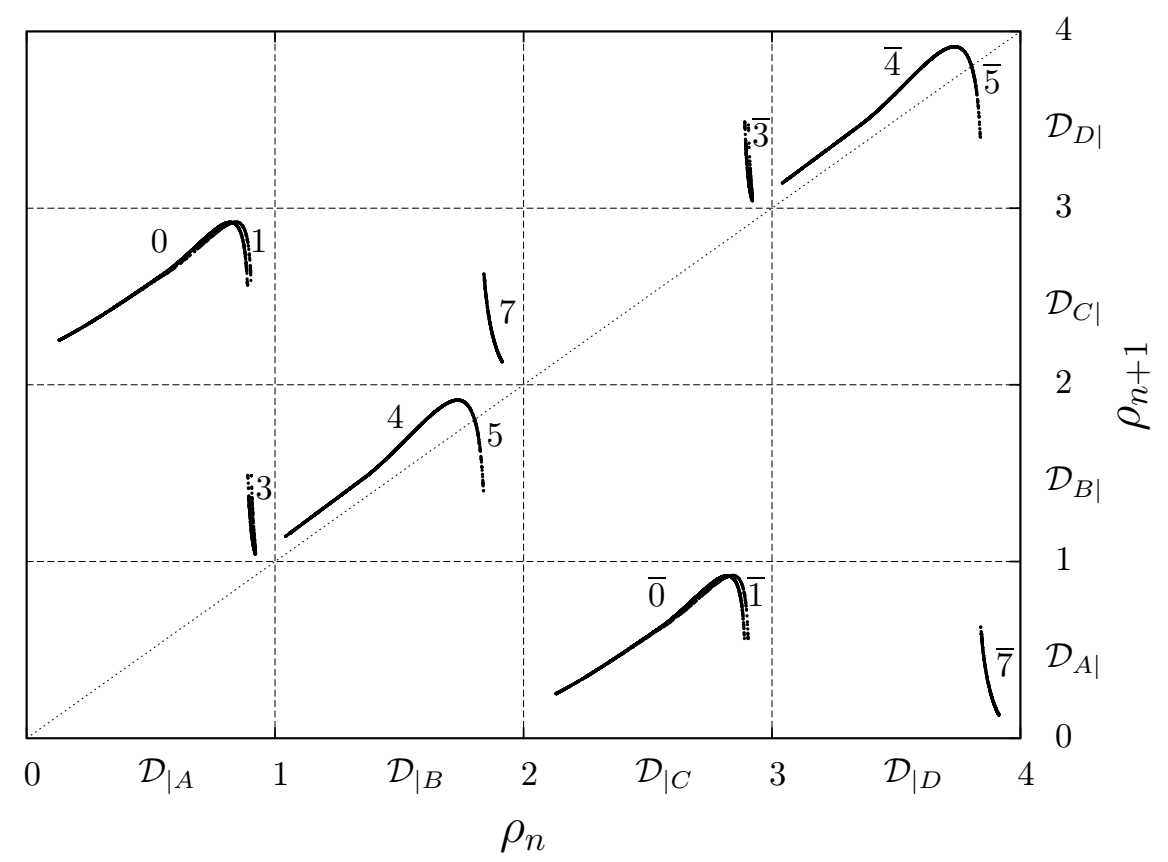

Fig. 14. First-return map to a four-component Poincaré section of the chaotic attractor solution to the equations (16). Parameter values are those of Fig. 12. 
In order to extract our template, we proceeded as follows. We constructed each inward open linking matrix independently. Let us start for instance with the domain $\mathcal{D}_{A \mid}=\{\overline{0}, \overline{1}, \overline{7}\}$. In this domain, we first determined torsions and permutations between branches " 0 " and " ")" which are both coming from component $\mathcal{C}_{C}$. Then, we determined torsions and permutations between branch $\overline{7}$ and the first two, " $\overline{0}$ " and "1". We proceeded in a similar way for domain $\mathcal{D}_{B \mid}=\{4,5,3\}$. Linking matrices $M_{C \mid}$ and $M_{D \mid}$ are then obtained by applying the procedure to get the image of a linking matrix $L_{\mid}$under an inversion symmetry [Rosalie \& Letellier, 2013], that is,

$$
\bar{L}_{\mid}=-L-\left|\begin{array}{ccc}
0 & +1 & +1 \\
+1 & 0 & +1 \\
+1 & +1 & 0
\end{array}\right|
$$

where $\bar{L}_{\mid}$is the symmetric of matrix $L \mid$ and $L$ is the corresponding open linking matrix [Rosalie \& Letellier, 2013]. We thus proposed the inward open linking matrices

$$
\left.\left.M_{A \mid}=\mid \begin{array}{ccc}
0 & -1 & -1 \\
-1 & -1 & -2 \\
-1 & -2 & -1
\end{array}\right], M_{B \mid}=\left|\begin{array}{ccc}
0 & -1 & -1 \\
-1 & -1 & -2 \\
-1 & -2 & -1
\end{array}\right|, M_{C \mid}=\mid \begin{array}{lll}
0 & 0 & 0 \\
0 & 1 & 1 \\
0 & 1 & 1
\end{array}\right] \text { and } M_{D \mid}=\left|\begin{array}{lll}
0 & 0 & 0 \\
0 & 1 & 1 \\
0 & 1 & 1
\end{array}\right|,
$$

where branches are ordered according the natural order in each domain. Matrix $M_{A \mid}\left(M_{B \mid}\right)$ is the symmetric matrix of $M_{C \mid}\left(M_{D \mid}\right)$ under an inversion symmetry as detailed in [Rosalie \& Letellier, 2013]. These four inward open mixers combined with the bounding torus induce a unique template (Fig. 15) and describe completely the topology of the multispiral attractor.

The template is then validated using linking numbers as for instance

$$
\begin{gathered}
l k(\overline{7} 0 \overline{0} 0 \overline{1} 34444447 \overline{0} 0 \overline{0} 0 \overline{0} 0 \overline{3} \overline{4} \overline{4} \overline{5}, \\
\overline{7} 0 \overline{0} 0 \overline{0} 0 \overline{1} 3444445447 \overline{0} 0 \overline{0} 0 \overline{3} \overline{4} \overline{4} \overline{4})=\frac{1}{2}(-41+11+3+9+6)=-6
\end{gathered}
$$

for which the oriented crossings were predicted using linking matrices (22). The orbits are segmented according to which domain the orbit visit. The oriented crossings between segments of different orbits are counted independently in each domain, and then summed. In each domain, the usual procedure as described by [Le Sceller et al., 1994] is applied using the "natural" order defined in each domain. From the elements of the linking matrices, we obtained a balance of 41 negative crossings and 11, 3, 9, and 6 positive crossings were induced by the joining charts of components $\mathcal{C}_{A}, \mathcal{C}_{B}, \mathcal{C}_{C}$, and $\mathcal{C}_{D}$, respectively. These two orbits were numerically extracted (Fig. 16) and the oriented crossings counted in the $x-y$ plane projection: we got -6 as predicted by our template.

\section{Summary and conclusions}

The topological analysis of attractors bounded by genus- $g$ bounding torus $A_{n}$ whose holes $(n$ being interior circles) are aligned, is as follows. First, for these tori, the genus is necessarily odd since $n=m+1$. There are $g-1$ components in the Poincaré section. The first-return map is computed from variable

$$
\rho_{n}=\sum_{i=1}^{g-1} \mathbb{I}_{\mathcal{C}_{i}}\left(i-1+\rho_{i, n}\right)
$$

where $i$ designates the $i$ th component. From the map, the $N \times N$ transition matrix $T_{a}$ is built, $N$ being the number of monotonic branches in the first-return map. The $(g-1)$ domains are thus determined with their natural orders. Inward open linking matrix are then determined and the template is drawn.

We thus proposed a general procedure to construct a template for attractors bounded by a bounding torus $A_{n}, A$ meaning that the interior holes are aligned, and $n$ being the number of interior circles which can be arbitrarily large. In order to do that, we enriched the concept of linking matrix to have an adequate description by $(g-1)$ linking matrices. By introducing a convention for representing clockwise the attractors, ordering the branches from the interior circles to the exterior boundary, and using the standard joining convention, the linking matrices induce an unique template. The case of attractors bounded by other types of torus (not necessarily aligned) is currently under considerations. 


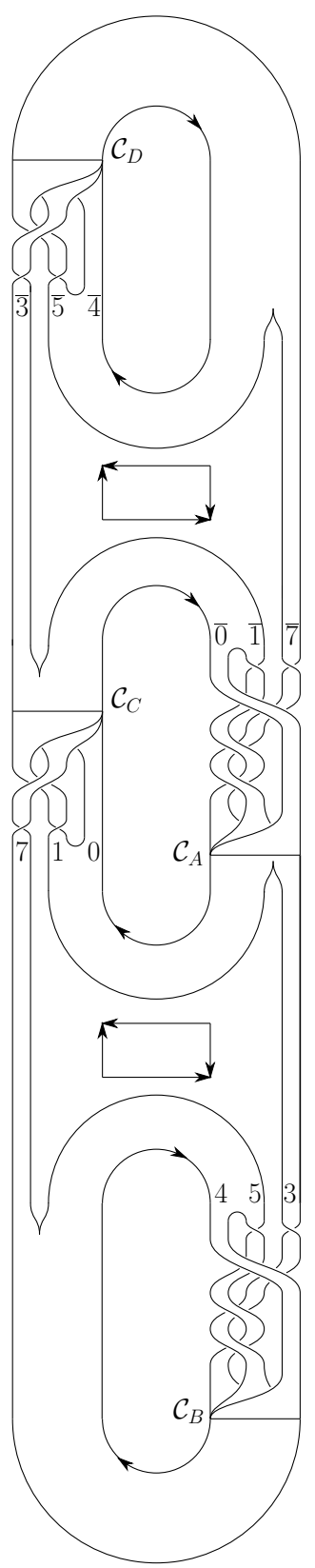

Fig. 15. Template that describes the attractor shown in Fig. 12.

\section{Acknowledgements:}

The authors thank Robert Gilmore for his helpful advice and comments.

\section{References}

A. Aziz-Alaoui [1999], "Differential equations with multispiral attractors", International Journal of Bifurcation and Chaos 9 (6), 1009-1039.

J. Birman \& R. Williams [1983], "Knotted periodic orbits in dynamical systems -I: Lorenz's equations", Topology, 22 (1), 47-82.

G. Byrne, R. Gilmore \& C. Letellier [2004], "Distinguishing between folding and tearing mechanisms in strange attractors", Physical Review E, 70, 056214.

J.-P. Eckmann, S. Oliffson Kamphorst, D. Ruelle \& S. Ciliberto [1986], "Lyapunov exponents from time 


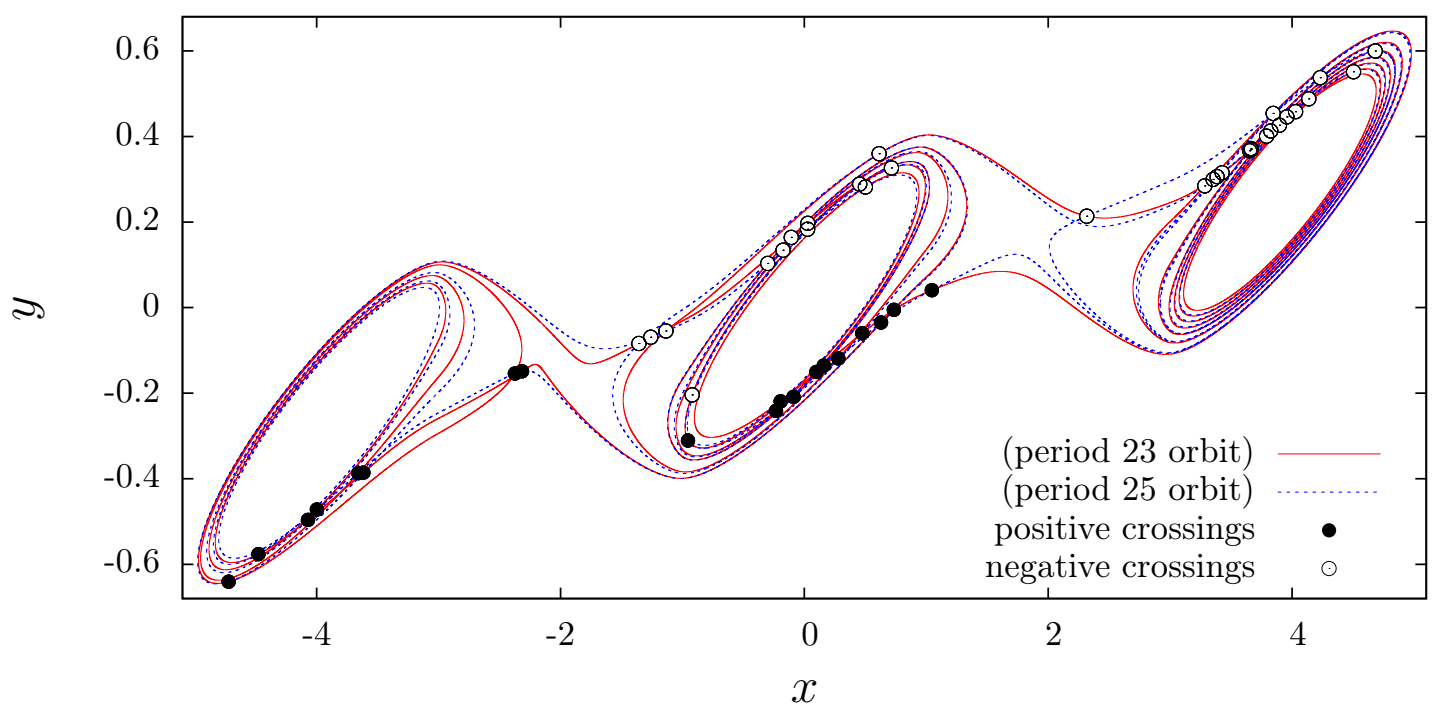

Fig. 16. Two unstable periodic orbits extracted from the chaotic multispiral attractor solution to equations (16). Their orbital sequences are $(\overline{7} 0 \overline{0} 0 \overline{1} 34444447 \overline{0} 0 \overline{0} 0 \overline{0} 0 \overline{3} \overline{4} \overline{4} \overline{5})$ and $(\overline{7} 0 \overline{0} 0 \overline{0} 0 \overline{1} 3444445447 \overline{0} 0 \overline{0} 0 \overline{3} \overline{4} \overline{4} \overline{4})$. The linking number between these two orbits is -6 . Parameter values are those of Fig. 12 .

series", Physical Review A, 34(6), 4971-4979.

R. W. Ghrist, P. J. Holmes \& M. C. Sullivan [1997], Knots and links in three-dimensional flows, (Book, Monographs and Lecture notes).

R. Gilmore \& M. Lefranc [2002], The topology of chaos, (Wiley).

R. Gilmore \& C. Letellier [2007], The symmetry of chaos, (Oxford University Press).

L. Glass [1999], "Chaos and heart rate variability", Journal of Cardiovascular Electrophysiology, 10, 13581360.

L. Glass [2009], "Introduction to controversial topics in nonlinear science: Is the heart rate chaotic?", Chaos, 19, 028501.

B. L. Hao [1989], Elementary Symbolic Dynamics and Chaos in Dissipative Systems, World Scientific Publishing, Singapore.

S. Jafari, J. C. Sprott \& S. M. R. H. Golpayegani [2013], "Elementary quadratic chaotic flows with no equilibria", Physical Letters A, 377, 699-702

L. Kocarev, Z. tasev \& D. Dimovski [1994], "Topological description of a chaotic atractor with spiral structure", Physics Letters A, 190, 399-402.

L. Le Sceller, C. Letellier \& G. Gouesbet [1994], "Algebraic evaluation of linking numbers of unstable periodic orbits in chaotic attractors", Physical Review E, 49 (5), 4693-4695.

C. Letellier, P. Dutertre \& B. Maheu [1995], "Unstable periodic orbits and templates of the Rössler system: toward a systematic topological characterization", Chaos, 5 (1), 271-282.

C. Letellier, P. Dutertre \& G. Gouesbet [1994], "Characterization of the Lorenz system taking into account the equivariance of the vector field", Physical Review E, 49 (4), 3492-3495.

C. Letellier \& G. Gouesbet [1996], "Topological characterization of reconstructed attractors modding out symmetries", Journal de Physique II, 6, 1615-1638.

C. Letellier, G. Gouesbet \& N. Rulkov [1996], "Topological analysis of chaos in equivariant electronic circuits", International Journal of Bifurcation \& Chaos, 6 (12B), 2531-2555.

C. Letellier \& R. Gilmore [2001], "Covering dynamical systems: Two-fold covers", Physical Review E, 63, 16206.

C. Letellier \& L. A. Aguirre [2012], "Required criteria for recognizing new types of chaos: Application to the "cord" attractor", Physical Review E, 85, 036204.

C. Letellier \& R. Gilmore [2013], "The universal template is a subtemplate of the double-scroll template", Journal of Physics A, 46 (6), 065102, 2013. 
E. N. Lorenz [1963], "Deterministic nonperiodic flow", Journal of the Atmospheric Sciences, 20, 130-141. H. Poincaré [1899], Les méthodes nouvelles de la mécanique céleste, Tome III (Gauthier-Villard, Paris).

M. Rosalie \& C. Letellier [2013] "Systematic template extraction from chaotic attractors. I genus-one attractors with an inversion symmetry", Journal of Physics A, Submitted.

O. E. Rössler [1976a], "Chaotic behavior in simple reaction system", Zeitschrift für Naturforschung A, 31, 259-264.

O. E. Rössler [1976b] "An equation for continuous chaos", Physics Letters A, 57 (5), 397-398.

T. D. Tsankov \& R. Gilmore [2003], "Strange attractors are classified by bounding tori", Physical Review Letters, 91 (13), 134104.

T. D. Tsankov \& R. Gilmore [2004], "Topological aspects of the structure of chaotic attractors in $\mathbb{R}^{3 "}$, Physical Review E, 69, 056206.

N. B. Tufillaro, T. Abbott \& J. Reilly [1992], An Experimental Approach to Nonlinear Dynamics and Chaos (Addison-Wesley, New York).

R. F. Williams [1977], "The structure of lorenz attractors", Lecture Notes in Mathematics, 615, 94-112.

A. Wolf, J. B. Swift, H. L. Swinney \& J. A. Vastano [1985], "Determining Lyapunov exponents from a time series", Physics D, 16, 285-317. 